\title{
Low Mass Gravitino: Re-Introducing the Superpartner as Dark Matter with Consideration to Inflation Due to Experimentation
}

\author{
AC Tahan ${ }^{1}$ \\ ${ }^{1}$ ETHERMED $_{\mathrm{Tм}}$, Cambridge, MA, USA \\ Correspondence: AC Tahan, ETHERMED, PO Box 391987, Cambridge, MA 02139, USA. E-mail: \\ actahan3@yahoo.com
}

Received: March 6, 2013

Accepted: October 9, $2013 \quad$ Online Published: November 12, 2013

doi:10.5539/mas.v7n12p43

URL: http://dx.doi.org/10.5539/mas.v7n12p43

\begin{abstract}
Superparticles including gravitons appearing in the laboratory due to a novel technique (Tahan, 2011, 2012) meant that the gravitino exists, concluded to have a low mass consequently requiring this affirmative presentation. A low mass gravitino would not have been problematic related to Big Bang Nucleosynthesis (BBN) and baryogenesis if understanding a correlation between inflation and gravitino abundance (Ellis, Linde, \& Nanopoulos, 1982), particularly when considering the particle to be dark matter and the lightest supersymmetric particle or superparticle (LSP). Gravitinos--proposed by this paper to be in the fifth dimension as dark matter--subsist because of inflation. This manuscript is a first discussion of the gravitino based on experimentation.
\end{abstract}

Keywords: Gravitino, supersymmetry, superparticles (superpartners), graviton, dark matter, D-brane, black hole, extra dimensions, entropy, inflation

\section{Background}

A novel technique that led to the observations of superpartner including graviton effects in the lab forced the need to present declaratively the existence of the gravitino, only speculative presentations being in the literature, and to propose anew how the low mass gravitino can exist as dark matter particularly in consideration of inflation, which has been appreciated as part of the history of the Cosmos though a specific particle related to it has not been observed. Since only inflation would have permitted an acceptable gravitino abundance in keeping with visualizations of the Universe (Khlopov \& Linde, 1984), the superpartner dark matter that accompanies the graviton can be acknowledged as a representative particle for it. Experienced readers could understand this work to be partly a review; yet, what should be remembered is that this manuscript is not a compendium regarding the gravitino but is focused on and exists due to the innovative Figure 1 method. Considering a varied readership due to interests in topics of this paper, well-recognized ideas to physicists are provided occasionally to improve understanding of information gathered experimentally. References are presented if related to what has been learned from experimentation; accordingly, certain readers may notice the omission of well-known citations that may have little connection to the experiments or have not been included since the works do not add significantly in relation to references already presented for the understanding of how the innovation can advance particular disciplines, e.g. dark matter studies.

Experiments were conducted to test the affects of low frequency quanta on Hydrogen in a magnetic field. From set-ups energy that imparted mass (mass-energy) emerged that could be directed over distances while adhering to the inverse square law, hinting at the possibility that the mass-energy was due to gravitons. Support for the emergence of the carrier graviton was observed when laser light was incorporated in the set-up for experiments as shown in Figure 1. The laser light should be appreciated not to have been part of the symmetry breaking technique but simply an addition of light to the set-up to understand if gravitons were emerging. The thought was that an increased bending of spacetime due to released gravitons from the Hydrogen area in the tube could change the path of the light traveling in spacetime--as with the bending of light by celestial bodies--because spacetime is ubiquitous, not only outside of the atmosphere of the Earth.

The light was recorded to curve around the tube holding the Hydrogen while a D-brane with an open string for the laser light appeared, which demonstrated that strings including the graviton exist thereby showing string theory to be a unifying theory (Tahan, 2011). The curving of the laser light resulted from gravitons having 
coupled to the tube, which consequently sufficiently bent spacetime due to additional mass from the mass-energy carrier gravitons. Based on the theoretical work for supergravity, the gravitino should be understood to exist due to the recorded effects of gravitons in the laboratory--the superpartner being well-accepted by scholars to accompany the boson graviton. Various control trials were performed to understand if the appearance of the D-brane that nearly mirrored images from literature of D-branes with open strings could be explained differently. No other conclusion in view of the set-up seemed reasonable, testable hypotheses resulting in being unacceptable.

\subsection{Introduction}

Consideration of the gravitino as dark matter (Pagels \& Primack, 1982) including in relation to inflation is unoriginal, as well as presenting the gravitino to be the LSP (Khlopov \& Linde, 1984; Bolz, Buchmüller, \& Plümacher, 1998; Moroi, Murayama, \& Yamaguchi, 1993); numerous other references could have been included since the gravitino has been well-studied theoretically, particularly as a dark matter particle. But the gravitino has never been known to exist. Various collider experiments have presented no evidence for supersymmetry, which would exclude the gravitino as dark matter particularly if superparticles are never detected when believing colliders to be the only means to study supersymmetry. Still, before the lack of supersymmetry evidence the gravitino had lost favor as a dark matter candidate, initially due to the gravitino problem (Weinberg, 1982). The Figure 1 technique allowing for examinations of supergravity permits conclusions no longer simply to be conceptual; this manuscript is unique since it discusses the gravitino as a reality. The facile, inexpensive method that allowed for symmetry breaking on the lab bench could permit direct studies of the gravitino, including other superparticles and strings.

Through experiments the nucleon was understood to be a brane that will be discussed in greater detail in upcoming manuscripts. The brane structure allowed for the picturing of a separation between the Standard Model visible sector and the underlying region of superparticles including the graviton. Accordingly, the symmetry breaking due to the Figure 1 method supported gauge mediated supersymmetry breaking (GMSB). In other words, by exposing Hydrogen in a specific magnetic field strength to particular low frequency quanta at a set amplitude, i.e. the Figure 1 technique, the underlying sector of superpartners was accessible through the quantized gauge field or SU(3) Yang-Mills theory: the Figure 1 method creating a symmetry breaking (Tahan, 2012) involving QCD. Experiments resulting in exposure of the Standard Model visible sector to superparticles including gravitons through GMSB meant that the gravitino should have a low mass.

After learning that a calculated mass for the Higgs boson using events involving the Figure 1 technique (Tahan, 2012) was not significantly different from synchrotron detections if the Standard Model Higgs boson has been found (Incandela, 2012; Gianotti, 2012), the gravitino mass was appreciated should not be over $1 \mathrm{keV}$, in consideration of theoretical work indicating that gravitinos would have overclosed the Universe otherwise (Cho \& Uehara, 2004) -- accepting the superpartner to be the LSP thereby preventing problems for BBN. Gravitinos being light supersymmetric particles would suggest the bodies to be dark matter (Takayama \& Yamaguchi, 2000; Giudice \& Rattazzi, 1999). Accordingly, superparticles can have sub-TeV masses while the gravitino can have a sub-keV mass (Albaid \& Babu, 2012), considerations that impelled this manuscript primarily since a sub-keV gravitino is not the consensus among scholars for the main dark matter candidate. Yet, other mass considerations will be mentioned in this manuscript for historical context and possibly for use by groups in future studies.

This paper does not suggest that scholars had stopped considering the gravitino in relation to the Cosmos. Though the gravitino grew less popular as a dark matter candidate, the influence of the superpartner also as the LSP has continued to be examined including for the particle make-up of the Universe, e.g. having impacted cosmic Lithium abundances (Bailly, Jedamzik, \& Moultaka, 2009). Emergence of the gravitino has been connected to baryogenesis (Trodden, 2004). Association of the superparticle with baryogenesis has been studied in detail, presentations having been nearly complete explanations with supersymmetry for baryogenesis and leptogenesis (Buchmüller, 1998). This manuscript can be included in discussions to bring nearer to completion work on baryogenesis since a problem in the field has been inclusion of the gravitino in view of its mass, i.e. uncertainty leading to theoretical studies with varied mass considerations as $<1 \mathrm{keV}$ or on the $\mathrm{TeV}$ level (Buchmüller, 1998). Accordingly, this manuscript as a confirmation of the existence of the gravitino should be understood necessary, events related to a low mass gravitino potentially answering multiple questions in the Cosmos. This work thus continues with an explanation of how the low mass gravitino can exist in the Universe, which involves inflation--existence of gravitino dark matter to have been a consequence of it. Discussion will include the potential influence entropy has had on the superparticle, particularly in relation to black holes due to observations of black hole evaporations in the laboratory (Tahan, 2011). The possible existence of primordial black holes as dark matter will be mentioned before describing the Figure 1 technique as a viable option for 
supersymmetry and string studies and concluding by reiterating certain observations from experiments, significant as contributions to explanations for the landscape of the Universe--presented by this manuscript to be a 2-brane.

\section{Gravitino Dark Matter and Inflation}

Observations in the laboratory suggesting the existence of a radion (graviscalar) field when the largest D-brane was recorded (Tahan, 2012) that will be discussed in an upcoming manuscript supported the existence of extra dimensions, the graviscalar being in the fifth dimension. The symmetry breaking due to the innovation that allowed for the emergences of superparticles including gravitons from the underlying region or fifth dimension created the thought that dark matter being a superparticle related to gravitons required re-assessment, particularly regarding how the dark matter could exist in the present Universe. The production of supergravity (Tahan, 2011, 2012) forced attention on the gravitino.

The LSP being a candidate for dark matter has been proposed, particularly that the body is the gravitino with a low mass $\mathrm{m}_{g_{3 / 2}} \lesssim 1 \mathrm{keV}$ (Pagels \& Primack, 1982). The gravitino has been argued should be stable due to R-parity conservation, which led to the suggestion that the long lifetime of the particle would be problematic, primarily regarding BBN (Weinberg, 1982) thereby causing the particle to lose popularity relative to subsequent proposals for dark matter. However, inflation was explained would resolve the gravitino problem, creating an abundance in keeping with BBN and the Cosmic Microwave Background (CMB) (Guth, 1981; Linde, Phys. Lett. 108B, 1982a; Linde, Phys. Lett. 114B, 1982b; Linde, Phys. Lett. 116B, 335, 1982c; Linde, Phys. Lett. 116B, 340, 1982d; Ellis, Linde, \& Nanopoulos, 1982). When supersymmetry was discovered could solve the gauge hierarchy problem, the prevailing mass of the gravitino became about $1 \mathrm{TeV}$. The higher mass did not fit well in explanations of the Universe, particularly that even a small number of decays would have prevented BBN or the thermal spectrum of the CMB (Dekel \& Ostriker, 1999). Yet, approximately $1 \mathrm{TeV}$ gravitinos were explained could exist without having been problematic for $\mathrm{BBN}$ and the $\mathrm{CMB}$ due to the reheating after inflation (Ellis, Kim, \& Nanopoulos, 1984).

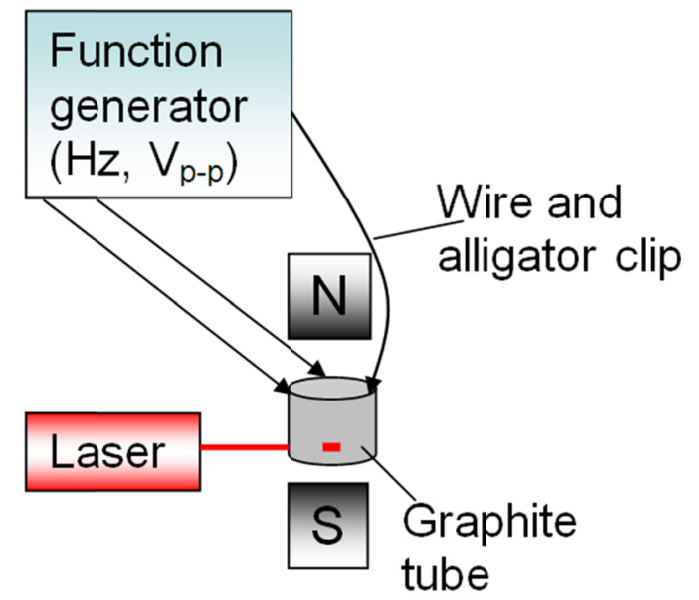

Figure 1. Set-up

96\% concd. Mallinckrodt Analytical Reagent, ACS sulfuric acid (typically $20 \mathrm{~mL}$ ) was placed in the graphite tube for each trial. The barometric pressure and relative humidity were about $99.7 \mathrm{kPa}$ and $98-100 \%$ (Boston, MA, CW1378) when the largest laser light D-brane was recorded. $\mathrm{N}$ and $\mathrm{S}$ are the poles of the gap magnet. The light from the laser (Quartet Standard Laser Pointer) was on the S pole side of the tube, about $.017 \mathrm{~m}$ from the center and .01-.02 m from the bottom. The positioning created an $\approx .004-.005 \mathrm{~m}$ line on the tube but did not prevent the light from entering a vacuum photocell that was connected to a digital oscilloscope. The photocell was used for positioning the laser; alignment allowed for consistent placement of the light on the tube. The same number of wooden blocks was placed under the photocell and the stand for the laser to raise the objects for alignment. The tube was clamped in the area of the static magnetic field measuring approximately $2000 \mathrm{Gs}$. Slight distance differences for the tube toward the $\mathrm{S}$ pole were not a factor; a distance between .004-.012 m should allow for observations. The laser was .34-.35 m from the tube. The low frequency examinations involved the maintaining of Hydrogen alignment in the magnetic field. Trials confirmed the importance of the magnet since results were not detected when it was eliminated. The function generator was connected to a LED on a breadboard from which wires provided $\approx 2 \mathrm{~Hz}(2.000-2.012 \mathrm{~Hz})$ and $\mathrm{V}_{\mathrm{p}-\mathrm{p}}=4.312-4.437$, predominately 4.375 , to 
the graphite tube. Only one wire with an alligator clip was needed, but three wires were attached to the top of the tube in a triangular formation to ensure that the frequency and amplitude were reaching it, particularly considering possible malfunction during a trial as due to corrosion of a clip. The laser was kept on by a clamp, and the batteries were changed before every trial to guarantee steadiness of the light. The photocell was .63-.64 $\mathrm{m}$ from the graphite tube, which was approximately $.098-.099 \mathrm{~kg}$. The clamp that held the tube was about .230 $\mathrm{kg}$, and the stand to which the clamp was attached was near $1.458 \mathrm{~kg}$, which included the $\approx .153 \mathrm{~kg}$ clamp holder. The photocell and oscilloscope were not part of the technique and were not needed if consistent light placement could be achieved without them, explaining why the items are not in the figure. The only parts always to be maintained are the frequency and amplitude, graphite tube (Crucible, Saed/Manfredi G40, 1.5"OD x 1.25"ID x 3.75 "DP), the consistent magnetic field strength (about $2000 \mathrm{Gs}$ ), and the concentrated sulfuric acid. Supplying the main components for the set-up differently can be tried, e.g. a Hydrogen source other than sulfuric acid. However, the other parts may have to be modified depending on the incorporation; for example, the amplitude may have to be adjusted. Changing the amplitude when using only one wire should not be needed. If the Figure 1 method is ineffective and the alligator clip of the wire is properly connected and not needing to be replaced, particularly if the experimenter had connected the three wires differently an amplitude adjustment for use with only the one wire may be needed considering that the three wires may have been providing quanta uniquely for proper string vibrations. The tube and acid should be replaced periodically to avoid variables, e.g. impurities in the acid or deformation of the tube over time due to the acid that could prevent consistent conduction of the frequency. The set-up allowed for the periodic emergences of superparticles including gravitons that can be written as a reaction: gluon, gluon, gluon, gluino, gluino, gluino, photon, proton, electron, $\mathrm{W}^{-}$boson, Higgs boson, $\mathrm{Z}$ boson, axion, dilaton $\rightarrow$ graviton, gravitino, neutron, electron neutrino, graviscalar, graviphoton, photon, photon, photon, sneutrino, selectron, saxion (Tahan, 2012). The reaction can be used to predict other particles and can be edited to include or eliminate bodies if determined necessary especially after incorporating proper detectors with the technique.

Figure 1 illustrates the set-up of the technique that allowed for the emergence of gravitons in the laboratory. The method involved utilizing $2 \mathrm{~Hz}$ at a specific amplitude to cause vibrations of the Hydrogen brane, which led to increased energy and consequently ultimately symmetry breaking that was periodic in the concentrated sulfuric acid Hydrogen source, i.e. symmetry breaking for different Hydrogen occurring at intervals during the typical approximately one hour trials. In other words, increasing string vibrations are equivalent to a growing energy (Scherk \& Schwarz, 1974). The $2 \mathrm{~Hz}$ was influential primarily since it affected gluon field strings: a strong force string underlying a field of quanta of a consistent nature (Yang \& Mills, 1954). The field strength (coupling constant) between quarks is uniform (Mills, 1994), and as the energy grew the coupling constant decreased (Aharony, Gubser, Maldacena, Ooguri, \& Oz, 2000). Since the Hydrogen stayed aligned in the magnetic field, the tension of the Hydrogen brane and associated strings remained relatively feeble thereby permitting the increasing vibrations. The proton, with consideration of electron capture (Tahan, 2012), resultantly eventually dissociated allowing for the glueball and symmetry breaking. Specifically, if understanding Yang-Mills theory to be a quantum gauge theory with the gauge group $\mathrm{SU}(\mathrm{N})$ being equal to a string theory with $1 / \mathrm{N}$ as the string coupling constant ('t Hooft, 1974; Maldacena, 1998), the strong force coupling constant gradually reached the limit 1/3, a large $\mathrm{N}$ analogous to a vibration limit (Aharony, Gubser, Maldacena, Ooguri, \& Oz, 2000), due to the continuous influence of the $2 \mathrm{~Hz}$ quanta that led to deconfinement. By that, the coupling constant can be thought inversely proportional to an energy that represents the gluon field. The growing oscillations of a string would cause a gluon field to approach a limit infinity ('t Hooft, 1974). At high energy from advancing to the limit, the coupling constant equaled zero that resulted in the release of gluon strings. The glueball consequently emerging due to the combining of the gluon strings (Tahan, 2012) prior to the massive superpartners of the graviton indicated that confinement and the Higgs mechanism are different facets of the strong force string (Fradkin \& Shenker, 1979). Accordingly, graviton-related observations in the laboratory (Tahan, 2011, 2012) were supportive of GMSB, which suggested the gravitino mass to be under $1 \mathrm{TeV}$ (Ratz, 2006).

What should be remembered is that conclusions in this manuscript are based on results from experiments. Observations occurred since the Figure 1 technique led to a symmetry breaking that exposed aspects of the Universe, appearances not being restricted to the lab. In other words, the emergences were from the ubiquitous Cosmos, the Figure 1 set-up only having been a means to experience previously conceptual unknowns. Accordingly, thoughts for the make-up of the Universe were unavoidable. For instance, gravitinos or other particle production that happened before inflation would have been extremely diluted by the expansion so that production would only have been of interest for history of the Universe studies. 
Gravitinos as dark matter thus emerged after inflation (Moroi, 1995). Inflatons, the inflaton field, after inflation decayed to particles and consequently released energy that increased the temperature of the Universe, i.e. the reheating after inflation. Slight R-parity violation likely occurred since stable R-parity could have permitted the production of additional gravitinos due to the decay of heavier superparticles. R-parity violation would have caused other superparticles to decay directly to Standard Model particles, not impacting the gravitino abundance (Bomark, 2011). With slight R-parity violation the gravitino as the LSP is unstable with a lifetime longer than the age of the Universe (Takayama \& Yamaguchi, 2000), stable on a cosmological timescale (Lemoine, Moultaka, \& Jedamzik, 2007).

Reheating is an important consideration since the topic has been connected directly to the gravitino problem. Reheating has been bothersome in discussions of the gravitino as dark matter since adjusting the reheating temperature as a function of the gravitino mass has been argued is required (Jedamzik, Lemoine, \& Moultaka, 2006). The relic abundance of gravitinos has been mentioned can be approximately linearly dependent on the reheating temperature permitting a gravitino density to account for dark matter (Bolz, Brandenburg, \& Buchmüller, 2001). But experimentation led to the conclusion that any discussions of the abundance should involve low mass gravitinos while considering the masses for other particles associated with the symmetry breaking, e.g. the Higgs boson, which is not extensively in the literature.

Scholars have explained that reheating need not be problematic if considering R-parity violation and the gravitino to be the LSP with a mass $\leqslant 1 \mathrm{keV}$. Specifically, creation of entropy has been suggested would cause necessary dilution of the gravitino abundance, though certain explanations have been insufficient. For instance, late decay of the lightest messenger particle to a visible sector particle thereby promoting entropy production, leading to dilution of the gravitino abundance, and resulting in suitable gravitino dark matter for randomly high reheating temperatures (Fujii \&Yanagida, 2002) has been argued to be an inadequate notion. At least two types of lightest messenger particles exist, and the lightest messenger particle with SU(2) quantum numbers would decay dominantly to vector bosons when the messenger number is broken. Accordingly, a problem would have been that the decays of messengers and superparticles would have occurred too quickly (Staub, Porod, \& Niemeyer, 2010; Staub, 2008).

Scenarios of entropy production have been proposed involving novel particles, e.g. the axion and saxion. However, the ideas (Baer, Kraml, Lessa, \& Sekmen, 2010; Kamada, Nakai, \& Sakai, 2011; Baer, Lessa, \& Sreethawong, 2012) have contained gravitinos that are too massive related to GMSB or have included a different Higgs boson mass from what has been suggested by experiments. Entropy production proposals allowing for an approximately $125 \mathrm{GeV}$ Higgs boson and low mass gravitinos (Ibe \& Sato, 2012) should be favored, particularly when including slight R-parity violation due to resulting advantages as not hindering BBN (Barbier et al., 2005; Pérez \& Spinner, 2012) -- the combination not having been found in the literature.

\subsection{Black Holes as Entropy Sources and the Universe Brane}

Black holes produced due to the Figure 1 technique and subsequent evaporations being observed (Tahan, 2011, 2012) suggested that a simple source of entropy in the Universe has been black holes. The holographic principle (holography) was suggested in the laboratory by the produced rotating black hole, a black hole D-brane, in the graphite tube providing entropy to the largest laser light D-brane recorded in trials and will be discussed in a future manuscript. Accordingly, black holes could be sources of entropy for the Universe brane, explaining Universe composition observations as the appearance of three-dimensional space ('t Hooft, 1993; Bekenstein, 1994; Susskind, 1995). Specifically, inflation often has been discussed separately from brane cosmology, particularly avoiding certain brane proposals that proponents of inflation have argued are problematic (Linde, 2002). Experimentation with the Figure 1 technique hinted at coexistence to some extent involving extra dimensions including D-branes. The inflation Universe is a 2-brane with a fifth dimension of superparticles including gravitons. The conclusion is an expansion of the understanding of nucleon branes and the observation of the D-brane with an open string (Tahan, 2011).

While primarily stable gravitino theoretical studies have concluded that late entropy production needed not occur in relation to a low mass gravitino $<\mathrm{keV}$ (Giudice \& Rattazzi, 1999), the understanding from research that black holes evaporate suggested that entropy potentially influenced the gravitino abundance in some regard, particularly if appreciating the possibility that primordial black holes existed (Carr \& Hawking, 1974). Theoretical studies have suggested that primordial black hole evaporations could have contributed entropy for the make-up of the Universe (Zel'dovich \& Starobinskiǐ, 1976; Frampton, 2009). Primordial black holes also have been discussed to be dark matter (Carr \& Hawking, 1974; Afshordi, McDonald, \& Spergel, 2003) including being all the dark matter in the Cosmos of the Standard Model (Frampton, Kawasaki, Takahashi, \& Yanagida, 
2010).

A conclusion from experimentation is that any discussions of dark matter must have supersymmetric considerations. Yet, since the reality of the fifth dimension was concluded from experiments the primordial black hole cannot be excluded as a dark matter candidate: theoretical work having suggested that extra dimensions could allow primordial black holes to subsist (Argyres, Dimopoulos, \& March-Russell, 1998). Accordingly, research with the Figure 1 technique suggested that multiple dark matter particles can exist.

Other dark matter bodies have been proposed in connection with the gravitino (Feng, Kamionkowski, \& Lee, 2010). Again this manuscript considers gravitinos as with other superparticles including the graviton to be in the underlying region or fifth dimension. Consequently, the primary dark matter being gravitinos and primordial black holes in the fifth dimension is plausible. The concurrence could be imagined to extend the existence of dark matter if considering the suggestion that primordial black hole evaporations could be sources of gravitinos (Khlopov, Barrau, \& Grain, 2006), apart from understanding that gravitinos could supplement primordial black hole dilutions. Thus, evaporation would have multiple purposes.

Entropy is considered to be provided to the Universe brane regardless of the gravitino. What was concluded from experimentation with the Figure 1 technique was that the D-branes for the laser light and produced black holes were in the fifth dimension. Therefore, expressing an entropy source for the Universe brane to be the fifth dimension is inclusive of black holes and vice versa unless a different entropy provider is mentioned in relation to the extra dimension.

Laser light D-branes were observed to be different sizes in trials, explained due to the black holes resulting from the Figure 1 technique in the graphite tube being energy sources. For example, the trial with the largest laser light D-brane seemed also to have the black hole with the greatest area, the appearances coinciding (Tahan, 2011, 2012). Observation of the nearly parallel positioning of the largest laser light D-brane relative to the graphite tube suggested that description of the fifth dimension as an underlying region should be reworded. Seemingly the bulk or fifth dimension surrounds or more specifically is ubiquitous in relation to the Universe brane, rather than visualizing it underneath. The Universe brane can be imagined resting in the bulk similarly to how an object would exist submerged in water. By that, as with other D-branes it is in the fifth dimension, i.e. all D-branes seem to be in the ubiquitous bulk or fifth dimension.

D-branes or for simplicity strings can be positioned in relation to the Universe brane thereby influencing the visible world, as with superparticles including gravitons. A black hole in the graphite tube had a specific orientation in relation to surroundings due to production. Thus, a brane would receive entropy or energy in relation to the position of the high gravitational area, which again was thought responsible for the observable dimensionality and size of the recorded, largest laser light D-brane. Accordingly, the extra dimension may provide entropy differently depending on the source, particularly in relation to the brane or object receiving the entropy.

The interaction of the black hole with the largest laser light D-brane was supportive of the high gravitational area having been a D0-brane or a collection of D0-branes, again concluded to be in the fifth dimension as with other D-branes. D0-branes have been described to act like gravitons (Banks, Fischler, Shenker, \& Susskind, 1997). The curving of the laser light around the graphite tube due to gravitons coupling to the tube was evidence for the bending of spacetime (Tahan, 2011). When understanding therefore that D0-branes and gravitons are in the fifth dimension, extreme warping or bending of the dimension should be considered. Addressed extensively in the literature, a warped extra dimension helps resolve long-standing cosmological problems: e.g. gauge and quark-lepton mass hierarchies (Hundi \& SenGupta, 2013; Das \& SenGupta, 2013). A warped fifth dimension permits visualization of string bodies to be positioned in relation to the Universe brane, for example superparticles including gravitons being near the brane in respect of the fifth dimension being infinite thus not needing a second brane to explain gravity (Randall, 2005; Randall \& Sundrum, 1999). Additionally, the focus of this paper on the fifth dimension should not be thought a disregard for compactified dimensions including Calabi-Yau space that will be mentioned in a future manuscript.

As suggested in experiments related to the produced black hole and the largest laser light D-brane, a more directional source of entropy for the three spatial dimensions would be logical. Accordingly as observed in trials regarding produced black holes and surroundings, relatively consistent orientation in regards to the Universe brane would support black holes to be the sources of entropy for three-dimensionality. Pretending that every black hole could evaporate in the lifetime of the Cosmos, naturally consequently a loss of three-dimensionality could be imagined. Thus, numerous scenarios for the existence of the Universe could be proposed including the eventual full elimination of three-dimensional space for the entire Universe 2-brane, particularly if other entropy 
as from the extra dimension would not compensate for the entropy lost from black holes. However with continued creation of entropy sources, e.g. black holes, including as the Universe expands uniform disappearance of all entropy is difficult to envision. Furthermore, a different entropy source may be found to be more similarly orientated to the Universe brane as the largest laser light D-brane and the associated black hole in the laboratory so that the entropy source or in combination to an entropy source as black holes may be determined to be more responsible for three-dimensionality.

The largest area black hole in the lab likely occurred due to interactions of gravitons with atoms in the tube, particularly considering that the production process of the Figure 1 technique was consistent so that black holes should not have been expected to be different sizes. Also, gravitons emerging from the fifth dimension and interacting thereby creating high gravitational areas, black holes, likely occurred particularly related to observed gravitational lensing of the laser light that will be discussed in an upcoming manuscript. In other words, gravitons influencing each other should be included in explanations for black hole observations in the laboratory and for the structures of black holes in the Universe especially if symmetry breaking might occur, thereby allowing for gravitons to emerge from the fifth dimension, due to the typical means of gravitational or stellar collapse that produces black holes in the Cosmos.

As taught in string theory that strings have tension (Zwiebach, 2004), experimentation supported that the Hydrogen brane has tension. Therefore when understanding superparticles including gravitons and branes to be in the fifth dimension, influence on the Universe brane would vary if concentrations of superparticles particularly gravitons were inconsistently positioned in relation to it. For instance, an additional cause for a black hole in the Cosmos could be an unusual amount of gravitons at a specific location of the fifth dimension near the Universe brane, i.e. concentrations of gravitons being observed as black holes in the dimensions of spacetime. Gravitons also should influence other particles including dark matter in the fifth dimension.

Entropy discussions in this manuscript have been related to the gravitino since a low mass gravitino has been proposed to be dark matter and the LSP. Yet, entropy likely played more roles at different periods of the Universe, including related to particle abundances. The most consistent result of entropy on the Universe brane historically seemingly would be the emergence of three-dimensional space. The description of the fifth dimension being ubiquitous may appear problematic regarding the providing of entropy to the Universe brane thereby resulting in three spatial dimensions. Thus, all entropy from the extra dimension seemingly would not contribute to the visible dimensionality of the Universe. Also, the discussion of entropy from the extra dimension should not be thought to eliminate contributions of entropy on the Universe brane.

\section{Colliders and the Figure 1 Technique}

Scholars have favored the scenario of late decay to have occurred in the Universe particularly when considering the gravitino to be the LSP and have suggested that detection of the gravitino might occur from next to lightest superparticle (NLSP) decay at facilities as synchrotrons. Since staff at synchrotrons continue to have difficulty detecting superparticles, the Figure 1 technique should be appreciated as a viable alternative for supersymmetry and string studies. The innovation has been the only method that has exposed supersymmetry and strings: e.g. the D-brane with an open string (Tahan, 2011).

The descriptions in this manuscript of low mass gravitinos, being dark matter and the LSP, support supersymmetry to subsist because of events related to inflation. Broken supersymmetry is appreciated to resolve problems with inflation and the landscape of the Universe (Linde, Phys. Lett. 116B, 335, 1982c; Ellis, Nanopoulos, Olive, \& Tamvakis, 1982; Ellis, Nanopoulos, Olive, \& Tamvakis, 1983). Accordingly, interdependence between supersymmetry and inflation can be stated to have been a reason for life, i.e. the Universe of Standard Model particles and dark matter would not exist otherwise. Supersymmetry breaking has not been more regularly discussed related to inflation because evidence for superparticles did not exist until the string experimentation with the Figure 1 set-up. The reheating after inflation would not have caused a return to the state before the broken symmetry (Linde, JETP Lett. 37, 1983b; Linde, Phys. Lett. 132B, 1983e; Nanopoulos, Olive, \& Srednicki, 1983). Also, symmetry breaking solves the primordial monopole problem; monopoles would not have appeared in the observable part of the Universe after the broken symmetry (Linde, Phys. Lett. 131B, 1983a).

Synchrotrons not having presented supersymmetry support it to be a broken symmetry, which allows superparticles to be heavier than corresponding Standard Model particles. Supersymmetry is broken in a hidden sector in GMSB (The ATLAS Collaboration 2012). Thus, the symmetry breaking due to the Figure 1 technique that allowed for access to the fifth dimension of superparticles (Tahan, 2011, 2012) can be thought a moving in an opposite direction regarding supersymmetry compared to at the beginning of the Universe. In other words, 
rather than shielding supersymmetry from the visible world the symmetry breaking exposed it.

The low mass and long lifetime of the gravitino could make detection with certain instruments difficult. The gravitino with a mass $\sim \mathrm{keV}$ has been suggested will not be observed in dark matter search experiments (Lemoine, Moultaka, \& Jedamzik, 2007). The weak coupling of the gravitino with matter makes direct detection with synchrotrons improbable. However, again scholars have suggested that colliders may provide data for gravitinos due to production from the decay of a NLSP that might create a variety of signals (Menci, Fiore, \& Lamastra, 2012). The experimental signature would depend on the nature of the NLSP (Aad et al., 2012). This manuscript supporting R-parity violation in the evolution of the Universe discourages the NLSP decay possibility. R-parity violation rules-out NLSP decay to gravitinos, the NLSP being short-lived and decaying directly to R-parity even particles (Takayama \& Yamaguchi, 2000), thereby reducing the chance that gravitinos can be detected at colliders and meaning that only the Figure 1 technique can allow for dark matter manifestations, apart from superparticles generally.

If the Figure 1 set-up would be placed in front of or preferably surrounded by a proper detector, measurement of low mass gravitinos could be argued would be supportive of the validity of chaotic inflation, apart from confirming the gravitino as dark matter. A detector could be focused primarily on the spin of the particle, though a direct mass measurement might be made. Yet if similar reasons for the inability to sight the gravitino with synchrotrons or specific dark matter experiments are encountered, success in having produced the gravitino can be understood simply with observations due to the production of the graviton, again the particle having been responsible for the recorded curving of the laser light around the tube in trials (Tahan, 2011). By that, evidence for the gravitino can be written has been seen, being the appearance of supergravity. Or an additional particle, e.g. a body as presented in the reaction of the Figure 1 description, could be detected that might be related to the gravitino, aside from superparticles emerging generally from the fifth dimension through use of the Figure 1 technique. The innovation could be incorporated for alternative studies, e.g. trying to trap and store the gravitino for analyses. Also, since a D-brane with an open string that was influenced by a produced high gravitational area was recorded (Tahan, 2011) use of the Figure 1 technique to see strings, apart from supersymmetry and dark matter, while incorporating a detector could advance studies considering that direct observation of a string is unlikely with the set-ups of present detectors or facilities. Therefore, as with the need for new technologies to study the unseen--e.g. the microscope having been needed to investigate the micro-world, the telescope having been required to examine phenomena outside of Earth, etc--the Figure 1 method should be understood the new tool for exploring science beyond the Standard Model.

\section{Conclusions}

Though experimentation proposed GMSB and a low mass gravitino, overall exclusion of other dark matter particles including bodies in the reaction of Figure 1 did not occur. Because trials confirmed the existence of extra dimensions this manuscript supports that multiple dark matter bodies could exist in the history of a Universe. Besides re-introducing the gravitino as dark matter this manuscript has specified that the gravitino should have a low mass, has indicated the validity of GMSB, has presented low mass gravitinos and primordial black holes to be dark matter, and has explained the participation of inflation for the existence of the low mass gravitino.

R-parity violation explains neutrino masses and mixings consequently resolving the atmospheric neutrino anomaly and solar neutrino problem (Romão, Díaz, Hirsch, Porod, \& Valle, 2000; Takayama \& Yamaguchi, 2000), numerous references could have been cited, that allows the gravitino as the LSP to be dark matter since the superpartner can be long-lived particularly compared to other superparticles (Takayama \& Yamaguchi, 2000). When remembering the black hole evaporations from trials and the thought that primordial black holes may presently exist due to extra dimensions, the primary dark matter thus being gravitinos in consideration of slight R-parity violation and primordial black holes is reasonable: gravitinos and primordial black holes being cold dark matter. Therefore, the Figure 1 method that led to the conclusion of a low mass gravitino provides answers to multiple cosmological questions.

Scholars have suggested that the dark matter was influential for the present make-up of the Cosmos, e.g. gravitinos having been involved possibly in the formation of galaxies (Efstathiou \& Silk, 1983). Black holes including evaporation also have been proposed could have created baryogenesis (Turner, 1979; Upadhyay, Gupta, \& Saxena, 1999) and contributed to the evolution of structures in the Universe, e.g. galaxy formation (Kawasaki, Kusenko, \& Yanagida, 2012; Carr, 1977). This manuscript has supported gravitinos to exist due to thermal production. The gravitino being $\lesssim 1 \mathrm{keV}$ is in keeping with theory regarding how best the particle could fit in the observable Universe. 
The proposal of other dark matter bodies is a probabilistic consideration due to the understanding of extra dimensions from the laboratory while appreciating the possibility of a multi-universe, i.e. different dark matter particles could be found depending on an environment or landscape though gravitinos and primordial black holes could account for all dark matter. Again supergravity was confirmed through experiments with the Figure 1 technique that created a symmetry breaking resulting in the emergences of superparticles including gravitons (Tahan, 2011, 2012). Chaotic inflation has been the only manner for implementing inflation in the context of supergravity (Goncharov \& Linde 1984; Linde, JETP Lett. 38, 1983c; Linde, Phys. Lett. 129B, 1983d). The appearance of the D-brane with an open string was supportive of chaotic inflation involving a multi-universe (Linde, 1986) -- a string theory landscape.

Again most literature is focused on the Cosmos of the Standard Model--fewer works incorporating supersymmetric dark matter particularly including extra dimensions and the Universe as a 2-brane. Laboratory work with the Figure 1 technique suggested the legitimacy of holography and exposed the radion (graviscalar) field of the fifth dimension that seemingly separated the black hole and laser light D-branes. The black hole D-brane having provided entropy to the largest laser light D-brane hinted that black holes in the Cosmos should be continuously providing entropy, which could be producing the appearance of three-dimensional space from the 2-brane.

Characteristics of the Universe brane as tension also have been proposed affected the abundances, including for the gravitino, and types of particles in the Cosmos (Mazumdar, 2001). Thus the distribution of gravitinos, entropy likely having participated and having contributed to the gravitino dark matter being cold (Lemoine, Moultaka, \& Jedamzik, 2007), might be suggested an insufficient explanation for gravitational influences typically associated with dark matter. The masses from present primordial black holes accordingly would compensate for the low mass of the gravitino; all dark matter bodies would contribute to nearby, characteristic events. Again other dark matter possibly accompanying the gravitino has been proposed, discussions having involved the gravitino as warm dark matter (Staub, Porod, \& Niemeyer, 2010). This work has been a first presentation suggesting gravitinos and primordial black holes could be cold dark matter simultaneously.

The recording of a D-brane with an open string and the understanding of the existence of an extra-dimension (Tahan, 2011, 2012) again was due to the Figure 1 method creating a symmetry breaking involving the Hydrogen brane, consequently causing superparticles including gravitons to emerge from the fifth dimension. Inclusions to the literature from this manuscript, a result of lab observations as superparticle effects, particularly related to the Universe brane with gravitino dark matter therefore should allow for a more complete picture of the Cosmos. Improved history discussions and organization of the Universe can be made by including fields, inflation, spontaneous supersymmetry breaking, extra dimensions particularly the fifth dimension, superparticles including gravitons, gravitinos as dark matter, R-parity violation, dark energy being vacuum energy, black hole evaporation, D-branes, and holography. This work should have contributed to the understanding of the interaction of components as mentioned in the previous sentence, including one factor influencing the existence of a different component, for the reality of the Universe.

\section{References}

Aad, G., Abbott, B., Abdallah, J., Abdel Khalek, S., Abdelalim, A. A., Abdesselam, A., ... Zwalinski, L. (2012). Search for events with large missing transverse momentum, jets, and at least two tau leptons in $7 \mathrm{TeV}$ proton-proton collision data with the ATLAS detector. Phys. Lett., 714B(2-5), 180-196, http://dx.doi.org/10.1016/j.physletb.2012.06.055

Afshordi, N., McDonald, P., \& Spergel, D. N. (2003). Primordial black holes as dark matter: The power spectrum and evaporation of early structures. ApJ, 594, L71-L74, http://dx.doi.org/10.1086/378763

Aharony, O., Gubser, S. S., Maldacena, J., Ooguri, H., \& Oz, Y. (2000). Large N field theories, string theory and gravity. Phys. Rep., 323, 183-386. http://dx.doi.org/10.1016/S0370-1573(99)00083-6

Albaid, A., \& Babu, K. S. (2012). Higgs boson of mass $125 \mathrm{GeV}$ in GMSB models with messenger-matter mixing. arXiv: 1207.1014v1 [hep-ph].

Argyres, P. C., Dimopoulos, S., \& March-Russell, J. (1998). Black holes and sub-millimeter dimensions. Phys. Lett., 441B, 96-104, http://dx.doi.org/10.1016/S0370-2693(98)01184-8

Baer, H., Kraml, S., Lessa, A., \& Sekmen, S. (2010) Thermal leptogenesis and the gravitino problem in the Asaka-Yanagida axion/axino dark matter scenario. arXiv: 1012.3769 [hep-ph].

Baer, H., Lessa, A., \& Sreethawong, W. (2012). Coupled Boltzmann calculation of mixed axion/neutralino cold dark matter production in the early universe. Journal of Cosmology and Astroparticle Physics, 36, 1-27, 
http://dx.doi.org/10.1088/1475-7516/2012/01/036

Bailly, S., Jedamzik, K., \& Moultaka, G. (2009). Gravitino dark matter and the cosmic lithium abundances. Physical Review D, 80(6). http://dx.doi.org/10.1103/PhysRevD.80.063509

Banks, T., Fischler, W., Shenker, S. H., \& Susskind, L. (1997). M Theory as a Matrix Model: A conjecture. Phys. Rev., 55D, 5112-5128, http://dx.doi.org/10.1103/PhysRevD.55.5112

Barbier, R., Berat, C., Besancon, M., Chemtob, M., Deandrea, A., Dudas, E., ... Sirois, Y. (2005). R-parity violating supersymmetry. Phys. Rept., 420, 1-202. http://dx.doi.org/10.1016/j.physrep.2005.08.006

Bekenstein, J. D. (1994). Entropy bounds and black hole remnants. Phys. Rev., 49D, 1912-1921. http://dx.doi.org/10.1103/PhysRevD.49.1912

Bolz, M., Buchmüller, W., \& Plümacher, M. (1998). Baryon asymmetry and dark matter. Phys. Lett., 443B, 209-213. http://dx.doi.org/10.1016/S0370-2693(98)01342-2

Bolz, M., Brandenburg, A., \& Buchmüller, W. (2001). Thermal production of gravitinos. Nucl. Phys., 606B, 518-544. http://dx.doi.org/10.1016/S0550-3213(01)00132-8

Bomark, N-E. (2011). Supersymmetry with trilinear R-Parity violation - Implications for cosmology and the LHC. Retrieved from http://hdl.handle.net/1956/5376

Buchmüller, W. (1998). Baryogenesis above the Fermi scale. DESY-98-171, 1-18, arXiv: hep-ph/9812447.

Carr, B. J., \& Hawking, S. W. (1974). Black holes in the early universe. Mon. Not. Roy. Astron. Soc., 168, 399-416.

Carr, B. J. (1977). The statistical clustering of primordial black holes. Astron. Astrophys., 56, 377-383.

Cho, Gi. C., \& Uehara, Y. (2004). Cosmological gravitino problem confronts electroweak physics. Phys. Rev., 69D, 075003. http://dx.doi.org/10.1103/PhysRevD.69.075003

Das, A., \& SenGupta, S. (2013). Gauge and moduli hierarchy in a multiply warped braneworld scenario. Phys. Lett., 718B, 1566-1570, http://dx.doi.org/10.1016/j.physletb.2012.12.056

Dekel, A., \& Ostriker, J. P. (1999). Formation of the structure of the universe. Cambridge: Cambridge University Press.

Efstathiou, G., \& Silk, J. (1983). The formation of galaxies. Fundamentals of Cosmic Physics, 9, 1-138.

Ellis, J., Linde, A. D., \& Nanopoulos, D. V. (1982). Inflation can save the gravitino. Phys. Lett., 188B, 59-64. http://dx.doi.org/10.1016/0370-2693(82)90601-3

Ellis, J., Nanopoulos, D. V., Olive, K. A., \& Tamvakis, K. (1982). Cosmological inflation cries out for supersymmetry. Phys. Lett., 118B, 335-339. http://dx.doi.org/10.1016/0370-2693(82)90198-8

Ellis, J., Nanopoulos, D. V., Olive, K. A., \& Tamvakis, K. (1983). Primordial supersymmetric inflation. Nucl. Phys., 221B, 524-548. http://dx.doi.org/10.1016/0550-3213(83)90592-8

Ellis, J., Kim, J. E., \& Nanopoulos, D. V. (1984). Cosmological gravitino regeneration and decay. Phys. Lett., 145B, 181-186. http://dx.doi.org/10.1016/0370-2693(84)90334-4

Feng, J. L., Kamionkowski, M., \& Lee, S. K. (2010). Light gravitinos at colliders and implications for cosmology. Phys. Rev., 82D, 015012. http://dx.doi.org/10.1103/PhysRevD.82.015012

Fradkin, E., \& Shenker, S. H. (1979). Phase diagrams of lattice gauge theories with Higgs fields. Phys. Rev., 19D, 3682-3697. http://dx.doi.org/10.1103/PhysRevD.19.3682

Frampton, P. H. (2009). Identification of all dark matter as black holes. JCAP, 10(16), 1-6. http://dx.doi.org/10.1088/1475-7516/2009/10/016

Frampton, P. H., Kawasaki, M., Takahashi, F., \& Yanagida, T. T. (2010). Primordial black holes as all dark matter. JCAP, 4(23), 1-10. http://dx.doi.org/10.1088/1475-7516/2010/04/023

Fujii, M., \& Yanagida, T. (2002). Natural gravitino dark matter and thermal leptogenesis in gauge-mediated $\begin{array}{lllll}\text { supersymmetry-breaking } \quad \text { models. } & \text { Phys. 273-283. }\end{array}$ http://dx.doi.org/10.1016/S0370-2693(02)02958-1

Gianotti, F. (2012). Update on the Standard Model Higgs searches in ATLAS, CERN Seminar.

Giudice, G. F., \& Rattazzi, R. (1999). Theories with gauge-mediated supersymmetry breaking. Phys. Rept., 322, 419-499. http://dx.doi.org/10.1016/S0370-1573(99)00042-3 
Goncharov, A. S., \& Linde, A. D. (1984). Chaotic inflation in supergravity. Phys. Lett., 139B, 27-30. http://dx.doi.org/10.1016/0370-2693(84)90027-3

Guth, A. H. (1981). Inflationary universe: A possible solution to the horizon and flatness problems. Phys. Rev., 23D, 347-356. http://dx.doi.org/10.1103/PhysRevD.23.347

Hundi, R. S., \& SenGupta, S. (2013). Fermion mass hierarchy in a multiple warped braneworld model. J. Phys. G: Nucl. Part. Phys., 40, 075002. http://dx.doi.org/10.1088/0954-3899/40/7/075002

Ibe, M., \& Sato, R. (2012). A $125 \mathrm{GeV}$ Higgs boson mass and gravitino dark matter in R-invariant direct gauge mediation. Phys. Lett., 717B, 197-201. http://dx.doi.org/10.1016/j.physletb.2012.09.018

Incandela, J. (2012). Update on the Standard Model Higgs searches in CMS, CERN Seminar.

Jedamzik, K., Lemoine, M., \& Moultaka, G. (2006). Gravitino dark matter in gauge mediated supersymmetry breaking. Phys. Rev., 73D, 043514. http://dx.doi.org/10.1103/PhysRevD.73.043514

Kamada, K., Nakai, Y., \& Sakai, M. (2011). Inflation in gauge mediation and gravitino dark matter. Prog. Theor. Phys., 125, 395-402. http://dx.doi.org/10.1143/PTP.125.395

Kawasaki, M., Kusenko, A., \& Yanagida, T. T. (2012). Primordial seeds of supermassive black holes. Phys. Lett., 711B, 1-5. http://dx.doi.org/10.1016/j.physletb.2012.03.056

Khlopov, M. Yu., Barrau, A., \& Grain, J. (2006). Gravitino production by primordial black hole evaporation and constraints on the inhomogeneity of the early universe. Class. Quant. Grav., 23, 1875-1882. http://dx.doi.org/10.1088/0264-9381/23/6/004

Khlopov, M. Yu., \& Linde, A. D. (1984). Is it easy to save the gravitino? Phys. Lett., 138B, 265-268. http://dx.doi.org/10.1016/0370-2693(84)91656-3

Lemoine, M., Moultaka, G., \& Jedamzik, K. (2007). Natural gravitino dark matter in SO(10) gauge mediated supersymmetry breaking. Phys. Lett., 645B, 222-227. http://dx.doi.org/10.1016/j.physletb.2006.12.025

Linde, A. D. (1982a). A new inflationary universe scenario: A possible solution of the horizon, flatness, homogeneity, isotropy and primordial monopole problems. Phys. Lett., 108B, 389-393. http://dx.doi.org/10.1016/0370-2693(82)91219-9

Linde, A. D. (1982b). Coleman-Weinberg theory and the new inflationary universe scenario. Phys. Lett., 114B, 431-435. http://dx.doi.org/10.1016/0370-2693(82)90086-7

Linde, A. D. (1982c). Scalar field fluctuations in the expanding universe and the new inflationary universe scenario. Phys. Lett., 116B, 335-339. http://dx.doi.org/10.1016/0370-2693(82)90293-3

Linde, A. D. (1982d). Temperature dependence of coupling constants and the phase transition in the Coleman-Weinberg theory. Phys. Lett., 116B, 340-342. http://dx.doi.org/10.1016/0370-2693(82)90294-5

Linde, A. D. (1983a). Inflation can break symmetry in SUSY. Phys. Lett., 131B, 330-334. http://dx.doi.org/10.1016/0370-2693(83)90510-5

Linde, A. D. (1983b). Supergravitation and the inflationary universe. JETP Lett., 37, 724-726.

Linde, A. D. (1983c). Chaotic inflating universe. JETP Lett., 38, 176-179.

Linde, A. D. (1983d) Chaotic inflation. Phys. Lett., 129B, 177-181. http://dx.doi.org/10.1016/0370-2693(83)90837-7

Linde, A. D. (1983e). Primordial inflation without primordial monopoles. Phys. Lett., 132B, 317-320. http://dx.doi.org/10.1016/0370-2693(83)90316-7

Linde, A. D. (1986). Eternal chaotic inflation. Mod. Phys. Lett., A1, 81-86. http://dx.doi.org/10.1142/S0217732386000129

Linde, A. (2002). Inflationary Theory versus Ekpyrotic/Cyclic Scenario. arXiv: hep-th/0205259.

Maldacena, J. (1998). The large N limit of superconformal field theories and supergravity. Adv. Theor. Math. Phys., 2, 231-252.

Mazumdar, A. (2001). Interesting consequences of brane cosmology. Phys. Rev., 64D, 027304. http://dx.doi.org/10.1103/PhysRevD.64.027304

Menci, N., Fiore, F., \& Lamastra, A. (2012). Galaxy formation in WDM Cosmology. arXiv: 1201.1617v2 [astro-ph.CO]. 
Mills, R. L. (1994). Space, time, and quanta: An introduction to contemporary physics, Part III. New York: W. H. Freeman.

Moroi, T., Murayama, H., \& Yamaguchi, M. (1993). Cosmological constraints on the light stable gravitino. Phys. Lett., 303B, 289-294, http://dx.doi.org/10.1016/0370-2693(93)91434-O

Moroi, T. (1995). Effects of the gravitino on the inflationary universe. arXiv: hep-ph/9503210.

Nanopoulos, D. V., Olive, K. A., \& Srednicki, M. A. (1983). After primordial inflation. Phys. Lett., 127B, 30-34. http://dx.doi.org/10.1016/0370-2693(83)91624-6

Pagels, H., \& Primack, J. R. (1982). Supersymmetry, cosmology, and new physics at teraelectronvolt energies. Phys. Rev. Lett., 48, 223-226, http://dx.doi.org/10.1103/PhysRevLett.48.223

Pérez, P. F., \& Spinner, S. (2012). The Minimal Theory for R-parity Violation at the LHC. JHEP 04, 118, 1-36, http://dx.doi.org/10.1007/JHEP04(2012)118

Randall, L. (2005). Warped passages: Unraveling the mysteries of the universe's hidden dimensions. New York: Harper Collins Publishers.

Randall, L., \& Sundrum, R. (1999). An alternative to compactification. Phys. Rev. Lett., 83, 4690-4693. http://dx.doi.org/10.1103/PhysRevLett.83.4690

Ratz, M. (2006). Theoretical aspects of gravitinos. Mini-workshop on Superweakly Interacting Dark Matter, DESY.

Romão, J. C., Díaz, M. A., Hirsch, M., Porod, W., \& Valle, J. F. W. (2000). A supersymmetric solution to the solar and atmospheric neutrino problems. Phys. Rev., 61D, 071703. http://dx.doi.org/10.1103/PhysRevD.61.071703

Scherk, J., \& Schwarz, J. H. (1974). Dual models for non-hadrons. Nucl. Phys., 81B, 118-144. http://dx.doi.org/10.1016/0550-3213(74)90010-8

Staub, F. (2008). Gauge Mediated SUSY Breaking with R-Parity Violation. Dark Matter at the Crossroads, DESY.

Staub, F., Porod, W., \& Niemeyer, J. (2010). Strong dark matter constraints on GMSB models. JHEP, 58, 1-14. http://dx.doi.org/10.1007/JHEP01(2010)058

Susskind, L. (1995). The world as a hologram. J. Math. Phys., 36, 6377-6396. http://dx.doi.org/10.1063/1.531249

't Hooft, G. (1974). A planar diagram theory for strong interactions. Nucl. Phys., 72B, 461-473. http://dx.doi.org/10.1016/0550-3213(74)90154-0

't Hooft, G. (1993). Dimensional reduction in quantum gravity. arXiv: gr-qc/9310026.

Tahan, A. C. (2011). Exposing strings in the laboratory with a novel technique. Appl. Phys. Res., 3(2), 39-51. http://dx.doi.org/10.5539/apr.v3n2p39

Tahan, A. C. (2012). Diagrammatic presentation for the production of gravitons and supersymmetry. Mod. Appl. Sci., 6(9), 76-83, http://dx.doi.org/10.5539/mas.v6n9p76

Takayama, F., \& Yamaguchi, M. (2000). Gravitino dark matter without r-parity. Phys. Lett., 485B, 388-392. http://dx.doi.org/10.1016/S0370-2693(00)00726-7

The ATLAS Collaboration. (2012). Search for physics beyond the Standard Model in events with a $Z$ boson and large missing transverse momentum using $\mathrm{s} \checkmark=7 \mathrm{TeV}$ pp collisions from the LHC with the ATLAS detector. ATLAS-CONF-2012-046.

Trodden, M. (2004). Baryogenesis and leptogenesis. XXXII SLAC Summer Institute, arXiv: hep-ph/0411301v1.

Turner, M. S. (1979). Baryon production by primordial black holes. Phys. Lett., 89B, 155-159. http://dx.doi.org/10.1016/0370-2693(79)90095-9

Upadhyay, N., Gupta, P. D., \& Saxena, R. P. (1999). Baryogenesis from primordial black holes after the electroweak phase transition. Phys. Rev., 60D, 063513. http://dx.doi.org/10.1103/PhysRevD.60.063513

Weinberg, S. (1982). Cosmological constraints on the scale of supersymmetry breaking. Phys. Rev. Lett., 48, 1303-1306. http://dx.doi.org/10.1103/PhysRevLett.48.1303

Yang, C. N., \& Mills, R. L. (1954). Conservation of isotopic spin and isotopic gauge invariance. Phys. Rev., 96, 
191-195. http://dx.doi.org/10.1103/PhysRev.96.191

Zel'dovich, Ya. B., \& Starobinskiǐ, A. A. (1976). Possibility of a cold cosmological singularity in the spectrum of primordial black holes. JETP Lett., 24, 571-573.

Zwiebach, B. (2004). A first course in string theory. Cambridge: Cambridge University Press. http://dx.doi.org/10.1017/CBO9780511841682

\section{Copyrights}

Copyright for this article is retained by the author(s), with first publication rights granted to the journal.

This is an open-access article distributed under the terms and conditions of the Creative Commons Attribution license (http://creativecommons.org/licenses/by/3.0/). 